\title{
NUMERICAL ANALYSIS OF DUSTY GAS FLOW IN A HYPERSONIC SHOCK TUNNEL
}

\author{
Yu. M. Tsirkunov and A. A. Verevkin \\ Baltic State Technical University, Faculty of Aerospace Engineering \\ 1 First Krasnoarmeiskaya Str., St. Petersburg 190005, Russia
}

\begin{abstract}
The results of a computational simulation of an unsteady two-phase gasparticle flow in a hypersonic shock tunnel with a convergent-divergent nozzle are described. In calculations, the configuration of the tunnel and the initial gas and particles' parameters were taken the same as in experiments carried out at the Central AeroHydroDynamic Institute (TsAGI) (Russia). The designed Mach number at the nozzle exit is $M=6.01$. Particles are injected into the high-pressure chamber of the shock tube just before the diaphragm opening. The carrier gas flow is described by the Euler equations which are solved by a finite-volume method of the second order. The particle-phase motion is simulated using the Lagrangian method. Developing in time a two-phase flow is investigated from the instant of opening the diaphragm between the high- and low-pressure chambers of the shock tube to the end of the quasi-steady-state flow over a model in the test section. Flow properties of both phases are discussed with emphasis on the problems that can appear in experiments.
\end{abstract}

\section{INTRODUCTION}

Shock tunnels are powerful tool in the experimental aerodynamics and physical gas dynamics. The potentialities of such tunnels and the theoretical prediction of the flow structure and temporal properties were the subjects of the intensive study in the midtwentieth century. The list of scientific publications on the theory and use of shock tunnels [1] contains about 700 such references. A hypersonic flow in the test section of these tunnels was achieved by combining a shock tube and a convergent-divergent nozzle. At that time, however, a direct numerical simulation of the flow in a hypersonic shock tunnel was impossible because of 
limitations of computers' capabilities. The first steps on the computational simulation of the gas flow in a shock tube with an adjacent convergent-divergent nozzle were made, apparently, in the very late 1960s (see, e.g., [2]). Since then, many separate aspects of the gas flow in a shock tube and in nozzles have been investigated numerically. During the last two decades, some important features of shock tunnel flows and starting processes in nozzles were simulated by computational fluid dynamics methods [3-6], but the flow in the whole tunnel from the instant of diaphragm opening up to the end of the quasi-steady-state flow regime in the test section was computed only in the very recent past [7]. The unsteady gas flow developing in such a shock tunnel after diaphragm opening has a very complex structure.

In recent years, hypersonic shock tunnels have been successfully used to study the dusty gas flow over bodies. The results of the research performed in the UT-1M experimental setup at TsAGI are the most well-known ones [8, 9]. Dispersed particles were injected into the high-pressure chamber of the shock tube just before the tunnel start-up (before diaphragm opening), they were entrained by the developing in time gas flow, reached the test section and interacted with a model. The general particle-phase flow structure near the model and the effect of particles on the heat flux at the model surface were investigated. However, no detailed data on the unsteady two-phase flow structure in the tunnel were obtained in those series of experiments. At the same time, it is clear that the particle-phase flow parameters in the test section depend on the flow history. Such phenomena as the particle velocity lag, particle-wall collisions, aerodynamic focusing of particles, etc. can be the causes of the particle redistribution in the flow, and hence, the nonuniformity of the particle-phase flow in the test section. The objective of this paper is to present and analyze the results of computational simulation of flows of both phases, the carrier gas and the particles, in a shock tunnel of UT-1M type from the instant of diaphragm opening to the end of the quasi-steady-state flow regime in the test section.

\section{SCHEMATIC OF A HYPERSONIC SHOCK TUNNEL AND INPUT PARAMETERS}

The computational simulation of two-phase gas-particle flow was carried out for the virtual shock tunnel shown schematically in Fig. 1. Geometrically, it is close to the real setup UT-1M at the TsAGI. The high-pressure and lowpressure chambers of the shock tube are cylindrical and of the same diameter as the diameter of the nozzle entrance. The length of the high-pressure chamber is $6 \mathrm{~m}$. The axially symmetrical contoured nozzle (de Laval nozzle) is designed for the exit Mach number of 6.01. Diameters of the entrance, throat 


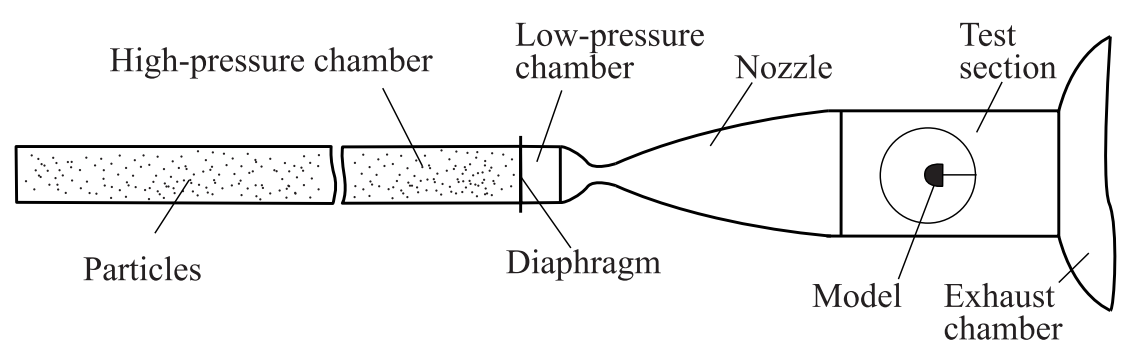

Figure 1 Schematic of a hypersonic shock tunnel

and exit of the nozzle are 76,38 , and $277.4 \mathrm{~mm}$, respectively. The length of the nozzle is $1.6 \mathrm{~m}$. The distance between the diaphragm and the throat of the nozzle is $0.4 \mathrm{~m}$. The diameter of the test section equals to that of the exit cross section of the nozzle. The model (sphere) is placed in the test section to which the exhaust chamber is attached. The diameter of the model is $20 \mathrm{~mm}$ and the distance between the exit cross section of the nozzle and the model is $250 \mathrm{~mm}$.

The initial parameters of the working gas (air) used in calculations, were the same as in experiments described in $[8,9]$. The temperature in the high-pressure chamber was $600 \mathrm{~K}$ (air was heated up to this temperature to prevent its condensation in the process of rarefaction in the nozzle and test section). The pressure in the high-pressure chamber was 25 bar. The pressure and the temperature in the other parts of the setup were 0.01 bar and $290 \mathrm{~K}$, respectively. The particles were assumed to be rigid spheres of equal radii. The silicon dioxide was taken as the particle material (its density is $\rho_{\mathrm{p}}=2264 \mathrm{~kg} / \mathrm{m}^{3}$ ). The particle diameter was varied from 0.15 to $40 \mu \mathrm{m}$ corresponding to a range of the characteristic Stokes number from $\sim 0.001$ to $\sim 10$. The smallest size of particles was less than the critical one, so that these particles did not reach the model in the test section and, hence, did not collide with its surface. The initial space distribution of particles in the high-pressure chamber was assumed to be random and uniform. The particle volume fraction in the high-pressure chamber was $\alpha_{\mathrm{p} \infty}=10^{-7}$ and the initial velocity of particles was zero.

\section{MATHEMATICAL MODEL OF THE TWO-PHASE FLOW}

Since the particle concentration in the flow is very low, the model of a one-way coupled two-phase flow model is used. This means that the effect of the dispersed phase on the carrier gas and the effect of particle-particle collisions are ignored. In this case, modeling of a gas-particle flow can be reduced to two sequential 
problems: modeling of the carrier gas flow without taking the dispersed phase into account, and the calculation of the particles' motion in the predetermined gas flow field.

The axially symmetric carrier gas flow is described by the Euler equations written in cylindrical coordinate system: the $x$-axis is directed along the axis of symmetry of the tunnel from the end of the high-pressure chamber towards the test section, $y$ is normal to it, $\phi$ is the azimuthal angle:

$$
\begin{gathered}
\frac{\partial \mathbf{U}}{\partial t}+\frac{\partial \mathbf{F}}{\partial x}+\frac{\partial \mathbf{G}}{\partial y}+\frac{\mathbf{H}}{y}=0 ; \\
\mathbf{U}=\left(\begin{array}{c}
\rho \\
\rho u \\
\rho v \\
\rho e
\end{array}\right) ; \quad \mathbf{F}=\left(\begin{array}{c}
\rho u \\
\rho u^{2}+p \\
\rho u v \\
(\rho e+p) u
\end{array}\right) ; \\
\mathbf{G}=\left(\begin{array}{c}
\rho v \\
\rho u v \\
\rho v^{2}+p \\
(\rho e+p) v
\end{array}\right) ; \quad \mathbf{H}=\left(\begin{array}{c}
\rho v \\
\rho u v \\
\rho v^{2} \\
(\rho e+p) v
\end{array}\right) ; \\
e=c_{v} T+\frac{u^{2}+v^{2}}{2} ; \quad p=\rho R T .
\end{gathered}
$$

Here, $t$ is the time; $\rho, p, e$, and $T$ are the density, the pressure, the total energy, and the temperature, respectively; $u$ and $v$ are the $x$ - and $y$-components of the velocity vector $\mathbf{v} ; c_{v}$ is the specific heat at a constant volume; and $R$ is the gas constant.

The domain of the flow under consideration includes the high-pressure and low-pressure chambers, the nozzle and the test section. The calculation domain in the $(x y)$-plane is bounded by the contour of the tunnel, the axis of symmetry, and the exit cross section of the test section. At the contours of the tunnel and the model, the condition of zero normal velocity is used. At the axis of symmetry, the velocity component $v$ and the derivatives $\partial \rho / \partial y, \partial T / \partial y$, and $\partial u / \partial y$ are taken equal to zero (the condition of symmetry). At the exit of the test section, no conditions are imposed.

The initial conditions (at $t=0$ ) were described above.

The dispersed phase flow is described by a Lagrangian formulation. The motion of a great number of individual particles is calculated. The model of action of the carrier gas on a particle includes the drag force $\mathbf{f}_{\mathrm{D}}$, the lift Magnus force $\mathbf{f}_{\mathrm{M}}$, and the damping torque $\mathbf{l}_{\mathrm{p}}$. The Magnus force is important for particles which get a high angular velocity when they bounce from the walls of the convergent part of the de Laval nozzle. Other effects of the gas-particle interaction and gravity are assumed to be negligible. The momentum and angular momen- 
tum equations, and the kinematic relation for the position-vector of a particle $\mathbf{r}_{\mathrm{p}}$ take the form:

$$
m_{p} \frac{d \mathbf{v}_{\mathrm{p}}}{d t}=\mathbf{f}_{\mathrm{D}}+\mathbf{f}_{\mathrm{M}} ; \quad J_{p} \frac{d \omega_{\mathrm{p}}}{d t}=\mathbf{l}_{\mathrm{p}} ; \quad \frac{d \mathbf{r}_{\mathrm{p}}}{d t}=\mathbf{v}_{\mathrm{p}}
$$

Here, $m_{\mathrm{p}}=(4 / 3) \pi \rho_{\mathrm{p}} r_{\mathrm{p}}^{3}, J_{\mathrm{p}}=(2 / 5) m_{\mathrm{p}} r_{\mathrm{p}}^{2}, \mathbf{v}_{\mathrm{p}}$, and $\omega_{\mathrm{p}}$ are the mass, the momentum of inertia, the velocity, and the angular velocity of a particle. The equations for $\mathbf{f}_{\mathrm{D}}, \mathbf{f}_{\mathrm{M}}$, and $\mathbf{l}_{\mathrm{p}}$ are usually written as follows:

$$
\begin{aligned}
\mathbf{f}_{\mathrm{D}} & =\frac{1}{2} C_{\mathrm{D}} \pi r_{\mathrm{p}}^{2} \rho\left|\mathbf{v}-\mathbf{v}_{\mathrm{p}}\right|\left(\mathbf{v}-\mathbf{v}_{\mathrm{p}}\right) \\
\mathbf{f}_{\mathrm{M}} & =\frac{4}{3} C_{\omega} \pi r_{\mathrm{p}}^{3} \rho\left[\left(\omega-\omega_{\mathrm{p}}\right) \times\left(\mathbf{v}-\mathbf{v}_{\mathrm{p}}\right)\right] \\
\mathbf{l}_{\mathrm{p}} & =\frac{1}{2} C_{l} r_{\mathrm{p}}^{5} \rho\left|\omega-\omega_{\mathrm{p}}\right|\left(\omega-\omega_{\mathrm{p}}\right)
\end{aligned}
$$

where $\omega=(1 / 2) \operatorname{curl} \mathbf{v}$.

The drag coefficient $C_{\mathrm{D}}$ was calculated from the approximation formulae proposed in [10]:

$$
C_{\mathrm{D}}=\left\{\begin{array}{lr}
C_{\mathrm{D}}^{1}, & 0 \leq \mathrm{M}_{\mathrm{p}} \leq 1 \\
C_{\mathrm{D}}^{12}, & 1<\mathrm{M}_{\mathrm{p}} \leq 1.75 \\
C_{\mathrm{D}}^{2}, & \mathrm{M}_{\mathrm{p}}>1.75
\end{array}\right.
$$

Here,

$$
\begin{gathered}
C_{\mathrm{D}}^{1}\left(\operatorname{Re}_{\mathrm{p}}, \mathrm{M}_{\mathrm{p}}, \frac{T_{\mathrm{p}}}{T}\right)=24\left\{\mathrm{Re}_{\mathrm{p}}\right. \\
\left.\quad+\sqrt{\frac{\gamma}{2}} \mathrm{M}_{\mathrm{p}}\left[4.33+\frac{3.65-1.53 T_{\mathrm{p}} / T}{1+0.353 T_{\mathrm{p}} / T} \exp \left(-0.247 \sqrt{\frac{2}{\gamma}} \frac{\operatorname{Re}_{\mathrm{p}}}{\mathrm{M}_{\mathrm{p}}}\right)\right]\right\}^{-1} \\
+\left[\frac{4.5+0.38\left(0.03 \mathrm{Re}_{\mathrm{p}}+0.48 \sqrt{\mathrm{Re}_{\mathrm{p}}}\right)}{1+0.03 \mathrm{Re}_{\mathrm{p}}+0.48 \sqrt{\mathrm{Re}_{\mathrm{p}}}}+0.1 \mathrm{M}_{\mathrm{p}}^{2}+0.2 \mathrm{M}_{\mathrm{p}}^{8}\right] \exp \left(-\frac{\mathrm{M}_{\mathrm{p}}}{2 \sqrt{\mathrm{Re}_{\mathrm{p}}}}\right) \\
C_{\mathrm{D}}^{2}\left(\operatorname{Re}_{\mathrm{p}}, \mathrm{M}_{\mathrm{p}}, \frac{T_{\mathrm{p}}}{T}\right)=\left[0.9+\frac{0.34}{\mathrm{M}_{\mathrm{p}}^{2}} \mathrm{M}_{\mathrm{p}}\left[1-\exp \left(-\frac{\mathrm{M}_{\mathrm{p}}}{\mathrm{Re}_{\mathrm{p}}}\right)\right]\right. \\
\left.+1.86 \sqrt{\frac{\mathrm{M}_{\mathrm{p}}}{\operatorname{Re}_{\mathrm{p}}}}\left(2+\frac{4}{\gamma \mathrm{M}_{\mathrm{p}}^{2}}+\frac{1.058}{\mathrm{M}_{\mathrm{p}}} \sqrt{\frac{2}{\gamma}} \sqrt{\frac{T_{\mathrm{p}}}{T}}-\frac{4}{\gamma^{2} \mathrm{M}_{\mathrm{p}}^{4}}\right)\right]\left(1+1.86 \sqrt{\frac{\mathrm{M}_{\mathrm{p}}}{\operatorname{Re}_{\mathrm{p}}}}\right)^{-1} ;
\end{gathered}
$$




$$
C_{\mathrm{D}}^{12}=C_{\mathrm{D} 1}^{1}+\frac{4}{3}\left(\mathrm{M}_{\mathrm{p}}-1\right)\left(C_{\mathrm{D} 2}^{2}-C_{\mathrm{D} 1}^{1}\right)
$$

where $\mathrm{M}_{\mathrm{p}}=\left|\mathbf{v}-\mathbf{v}_{\mathrm{p}}\right| / \sqrt{\gamma R T}$ and $\operatorname{Re}_{\mathrm{p}}=2 \rho\left|\mathbf{v}-\mathbf{v}_{\mathrm{p}}\right| r_{\mathrm{p}} / \mu$ are the particle Mach and Reynolds numbers; $C_{\mathrm{D} 1}^{1}$ is the value of $C_{\mathrm{D}}^{1}$ at $\mathrm{M}_{\mathrm{p}}=1$; and $C_{\mathrm{D} 2}^{2}$ is the value of $C_{\mathrm{D}}^{2}$ at $\mathrm{M}_{\mathrm{p}}=1.75$.

In the flow under consideration, the dependence of $C_{\mathrm{D}}$ on $T_{\mathrm{p}} / T$ is very weak; so, it was ignored and the ratio $T_{\mathrm{p}} / T$ was taken equal to unity.

For calculation of $C_{\omega}$, the exact solution from [11] and the formula proposed in [12] were used:

$$
C_{\omega}= \begin{cases}\frac{3}{4}, & 2 \gamma_{\omega}<0.45 \\ \frac{3}{8} \hat{C}_{\omega}, & 2 \gamma_{\omega} \geq 0.45\end{cases}
$$

where

$$
\gamma_{\omega}=\frac{\left|\omega-\omega_{\mathrm{p}}\right| r_{\mathrm{p}}}{\left|\mathbf{v}-\mathbf{v}_{\mathrm{p}}\right|} ; \quad \hat{C}_{\omega}\left(\gamma_{\omega}, \operatorname{Re}_{\mathrm{p}}\right)=\gamma_{\omega}^{-1}\left[0.45+\left(2 \gamma_{\omega}-0.45\right) \exp \left(-0.075 \gamma_{\omega}^{0.4} \operatorname{Re}_{\mathrm{p}}^{0.7}\right)\right] \text {. }
$$

The expression for the coefficient $C_{l}$ was taken in the form proposed in [13]:

$$
C_{l}=\frac{C_{l 1}}{\sqrt{\operatorname{Re}_{\mathrm{p} \omega}}}+\frac{C_{l 2}}{\operatorname{Re}_{\mathrm{p} \omega}}
$$

Table 1 Coefficients $C_{l 1}$ and $C_{l 2}$ in different ranges of the particle rotational Reynolds number $\operatorname{Re}_{\mathrm{p} \omega}$

\begin{tabular}{ccc}
\hline $\mathrm{Re}_{\mathrm{p} \omega}$ & $C_{l 1}$ & $C_{l 2}$ \\
\hline $0-6$ & 0 & $16 \pi$ \\
$6-20$ & 5.32 & 37.2 \\
$20-50$ & 6.44 & 32.2 \\
$50-4 \cdot 10^{4}$ & 6.45 & 32.1 \\
\hline
\end{tabular}

where $\operatorname{Re}_{\mathrm{p} \omega}=\rho\left|\omega-\omega_{\mathrm{p}}\right| r_{\mathrm{p}}^{2} / \mu$, and constants $C_{l 1}$ and $C_{l 2}$ are given in Table 1.

The boundary conditions for particles depend on the boundary type. If a particle crosses the axis of symmetry, we use the condition of specular reflection from the axis. If a particle crosses the exit cross section of the test section, it is excluded from further consideration. If a particle collides with the walls of the tunnel or the model, its rebound is considered as frictional and not completely elastic, and the semiempirical particlewall collision model developed in [14] is used for calculating the parameters of a particle just after its rebound. This model is based on the laws of mechanics and the experimental data [15] for the restitution coefficients of the normal and tangential to the wall velocity components of the particle gravity center. This model is valid at moderate and high particle impact velocities. The final relations for the normal, tangential, and angular velocities of a particle after its rebound take the form:

$$
v_{\mathrm{p} n}^{+}=-a_{n} v_{\mathrm{p} n}^{-} ; \quad v_{\mathrm{p} \tau}^{+}= \begin{cases}a_{\tau} v_{\mathrm{p} \tau}^{-}+\omega_{\mathrm{p}}^{-} r_{\mathrm{p}}\left(a_{\tau}-1\right), & \beta<\beta_{*} \\ a_{\tau} v_{\mathrm{p} \tau}^{-}-\frac{2}{7} \omega_{\mathrm{p}}^{-} r_{\mathrm{p}}, & \beta \geq \beta_{*}\end{cases}
$$




$$
\omega_{\mathrm{p}}^{+}= \begin{cases}\frac{5}{2} \frac{v_{\mathrm{p} \tau}^{-}}{r_{\mathrm{p}}}\left(a_{\tau}-1\right)+\frac{5}{2} \omega_{\mathrm{p}}^{-}\left(a_{\tau}-\frac{3}{5}\right), & \beta<\beta_{*} ; \\ -\frac{v_{\mathrm{p} \tau}^{-}}{r_{\mathrm{p}}} a_{\tau}+\frac{2}{7} \omega_{\mathrm{p}}^{-}, & \beta \geq \beta_{*} .\end{cases}
$$

Here, $v_{\mathrm{p} n}^{-}, v_{\mathrm{p} \tau}^{-}$, and $\omega_{\mathrm{p}}^{-}$are the normal, tangential, and angular velocities of a particle just before its collision; $a_{n}$ and $a_{\tau}$ are the restitution coefficients; $\beta$ is the collision angle (the angle between the velocity vector of a particle before a collision $\mathbf{v}_{\mathrm{p}}^{-}$and the wall); and $\beta_{*}$ is the critical value of $\beta$ : if $\beta<\beta_{*}$, the collision is considered to be sliding, if $\beta \geq \beta_{*}$, a collision is considered as nonsliding.

For calculation of the restitution coefficients, the following formulae were used $[14,15]$ :

$$
\begin{aligned}
& a_{n}=1-\left\{1-\exp \left[-0.1\left(V_{\mathrm{p}}^{-}\right)^{0.61}\right]\right\} \sin \beta ; \quad V_{\mathrm{p}}^{-}=\left[\left(v_{\mathrm{p} n}^{-}\right)^{2}+\left(v_{\mathrm{p} \tau}^{-}\right)^{2}\right]^{1 / 2} \\
& a_{\tau}=C_{0}+C_{1}(\hat{\beta})^{2}+C_{2}(\hat{\beta})^{4}+C_{3}(\hat{\beta})^{6} ; \quad \hat{\beta}=\frac{\pi}{2}-\beta
\end{aligned}
$$

The coefficients in the last formula and the critical angle $\beta_{*}$ depend on the wall and particle materials. In calculations, they were taken as follows: $C_{0}=0.690$, $C_{1}=-0.288, C_{2}=0.1140, C_{3}=0.0219$, and $\beta_{*}=0.1911$. These values correspond to particles of hard materials like corundum or silicon dioxide, and a carbon steel (like C45) wall.

At the instant of the start-up of the shock tube $(t=0)$, the particles in the high-pressure chamber are assumed to be suspended in the carrier gas, and their translational and angular velocities are taken equal to zero.

\section{NUMERICAL METHOD}

For solving the Euler equations, a finite-volume method was used. In the calculational domain, the unstructured triangular grid was generated with a high concentration of nodes in the nozzle throat and near the model. This grid was used to construct the mesh of polygonal cells with centers at the vertices of triangles. Total number of cells was 325,000 for regular calculations and 788,000 for preliminary tests. The Godunov scheme [16] of the second-order in space and time was used for the computation of the unsteady flow. Simultaneously with simulating the carrier gas flow, Eqs. (1) were calculated for a large number of particles using the second-order predictor-corrector method. Parameters of the carrier gas at the point of the particle location were calculated using the linear interpolation of the gas parameters taken at the nodes of the triangle element of the grid in which a particle is placed at a given time. 
The time step $\Delta t$ in the computational algorithm for the two-phase flow was taken as a minimal value of a time step $\Delta t_{g}$ calculated from the CourantFriedrichs-Levy condition for the explicit finite-volume scheme applied to the Euler equations, and of a time step $\Delta t_{\mathrm{p}}$ in the above equations for a particle. The latter was limited by a distance of particle traveling during $\Delta t_{\mathrm{p}}$ which had to be less than the radius of a circle inscribed in the triangle element.

In parallel with the calculation of particle trajectories, the profiles of the particle volume fraction were calculated in the nozzle and in the test section before the model. For this, the dispersed phase was treated as a set of a large number of simulated particles. Each simulated particle represented a cloud of real particles placed in its vicinity. The total number of simulated particles was about $2 \cdot 10^{6}$. For further details of the numerical algorithm, see $[17]$.

\section{DISCUSSION OF RESULTS}

The application of a shock tunnel for an experimental study of a dusty gas flow over a body is based on the default assumptions that the two-phase gasparticle flow in the test section is uniform and steady-state, at least during a short time interval (normally, several milliseconds) which is enough for taking measurements. However it is clear that the particle-phase flow parameters in the test section depend on the behavior of particles in the developing unsteady gas flow, and such behavior can be very complex. The results of numerical investigation of flows of the carrier gas and the particles in the shock tunnel (see Fig. 1) are presented and discussed below.

The carrier gas flow visualization was performed in two ways: as a field of Mach number and as patterns of massless particles-markers. The latter way allowed tracing the gas motion, in particular the evolution of contact discontinuities shape.

\subsection{Flow in the Nozzle}

Just after opening of the diaphragm, the rarefaction wave propagates to the left end of the high-pressure chamber (see Fig. 1) and the primary shock wave (PSW) travels towards the nozzle throat and interacts with the nozzle walls. This process is accompanied by arising of the transverse shock waves which interact with each other regularly and irregularly, as it is illustrated by Fig. 2. The contact discontinuity (CD) separating gases initially located in high-pressure and lowpressure chambers of the shock tube also travels to the nozzle and interacts with 


\section{$\mathrm{M}$}

\section{$\begin{array}{llllllllll}0.00 & 0.54 & 1.09 & 1.63 & 2.18 & 2.72 & 3.27 & 3.81 & 4.34 & 4.90\end{array}$}

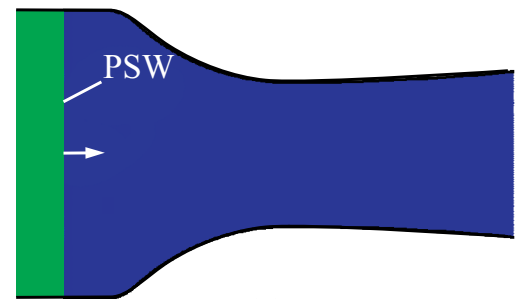

(a)

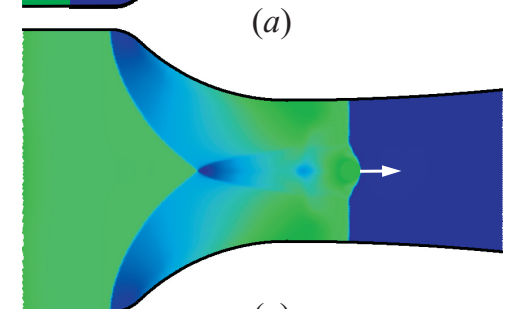

(c)

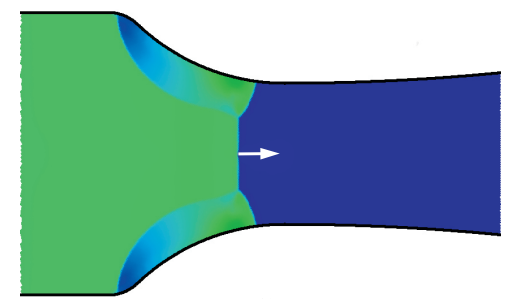

(b)

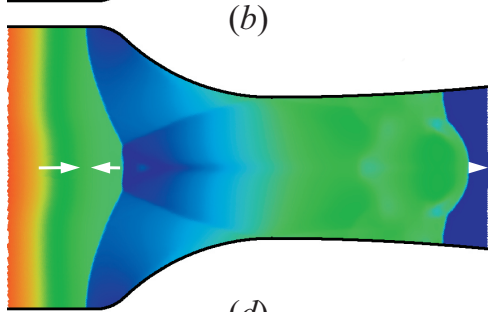

(d)
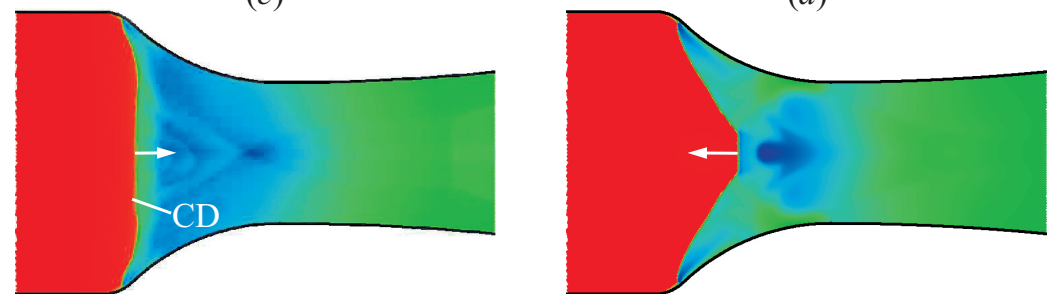

(e)
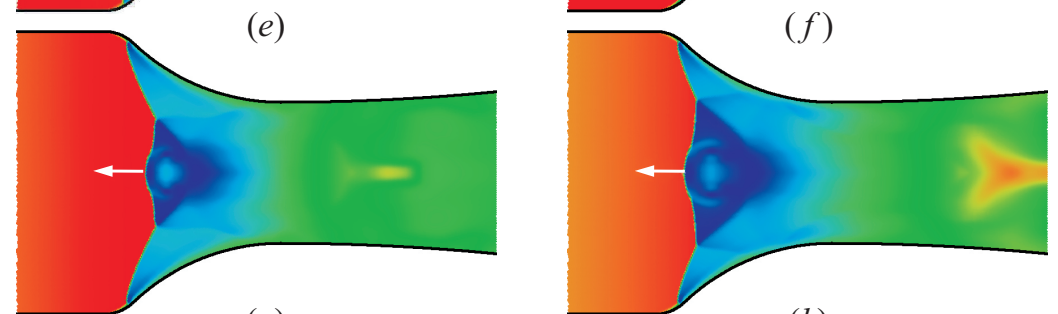

$(g)$
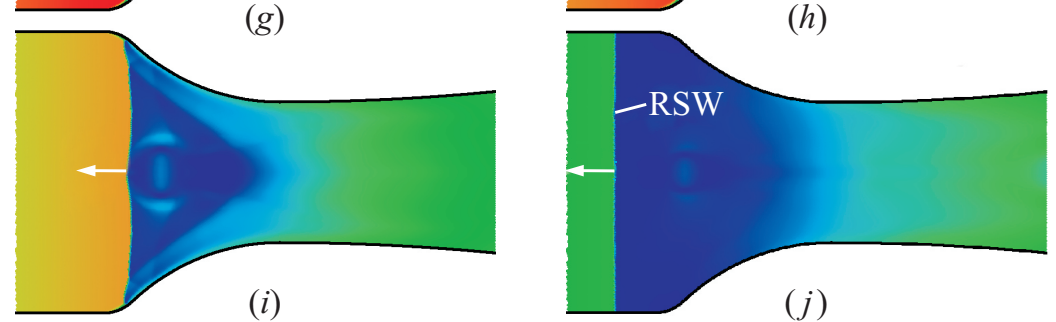

Figure 2 Field of Mach number in the nozzle throat at different $t$ : (a) $0.22 \mathrm{~ms}$; (b) 0.25 ; (c) $0.264 ;(d) 0.28$; (e) $0.31 ;(f) 0.34 ;(g) 0.38$; $(h) 0.4 ;(i) 0.444$; and (j) $0.6 \mathrm{~ms}$. 
M

$\begin{array}{llllllllll}0.00 & 0.54 & 1.09 & 1.63 & 2.18 & 2.72 & 3.27 & 3.81 & 4.34 & 4.90\end{array}$
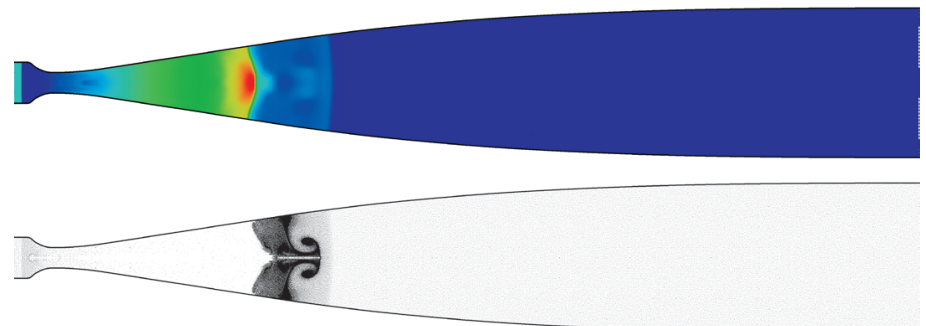

(a)
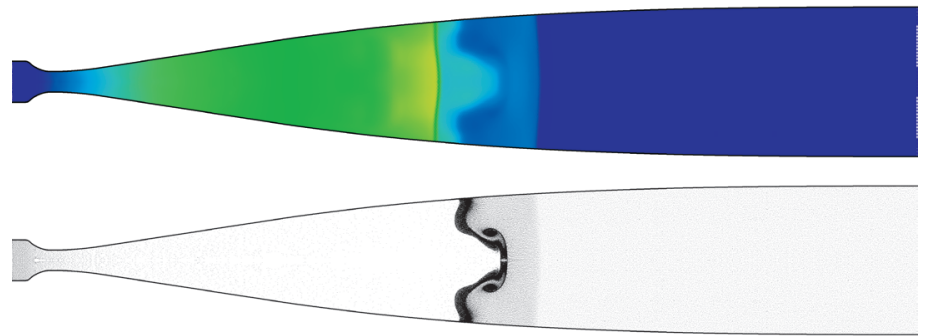

(b)
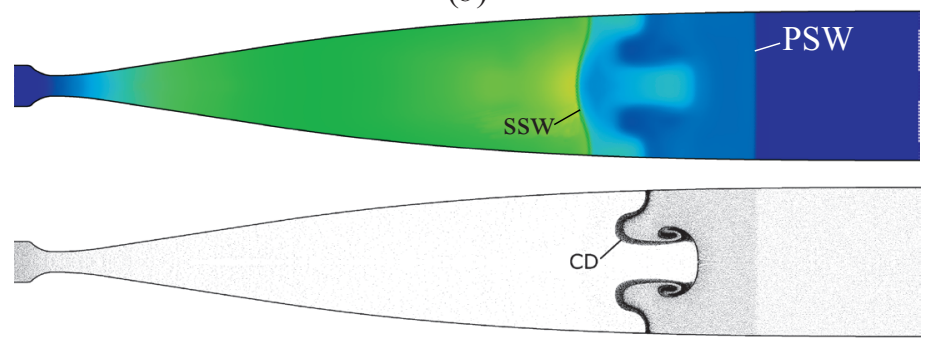

(c)
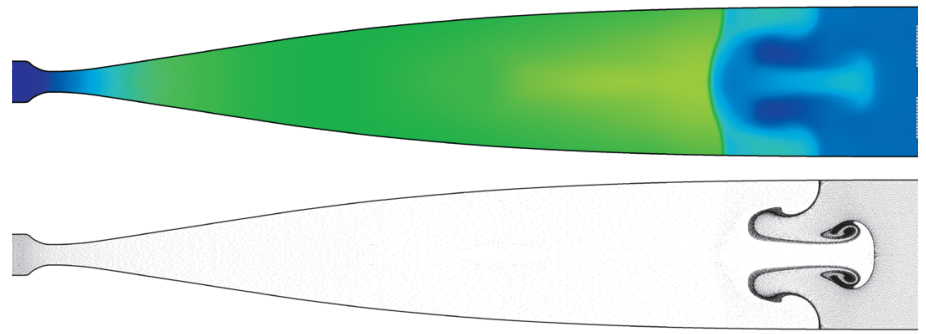

(d)

Figure 3 Field of Mach number and corresponding to it instant pattern of massless particles-markers in the divergent part of the nozzle at different $t:(a) 0.6 \mathrm{~ms} ;(b) 1$; (c) 1.4; and (d) $1.8 \mathrm{~ms}$. 


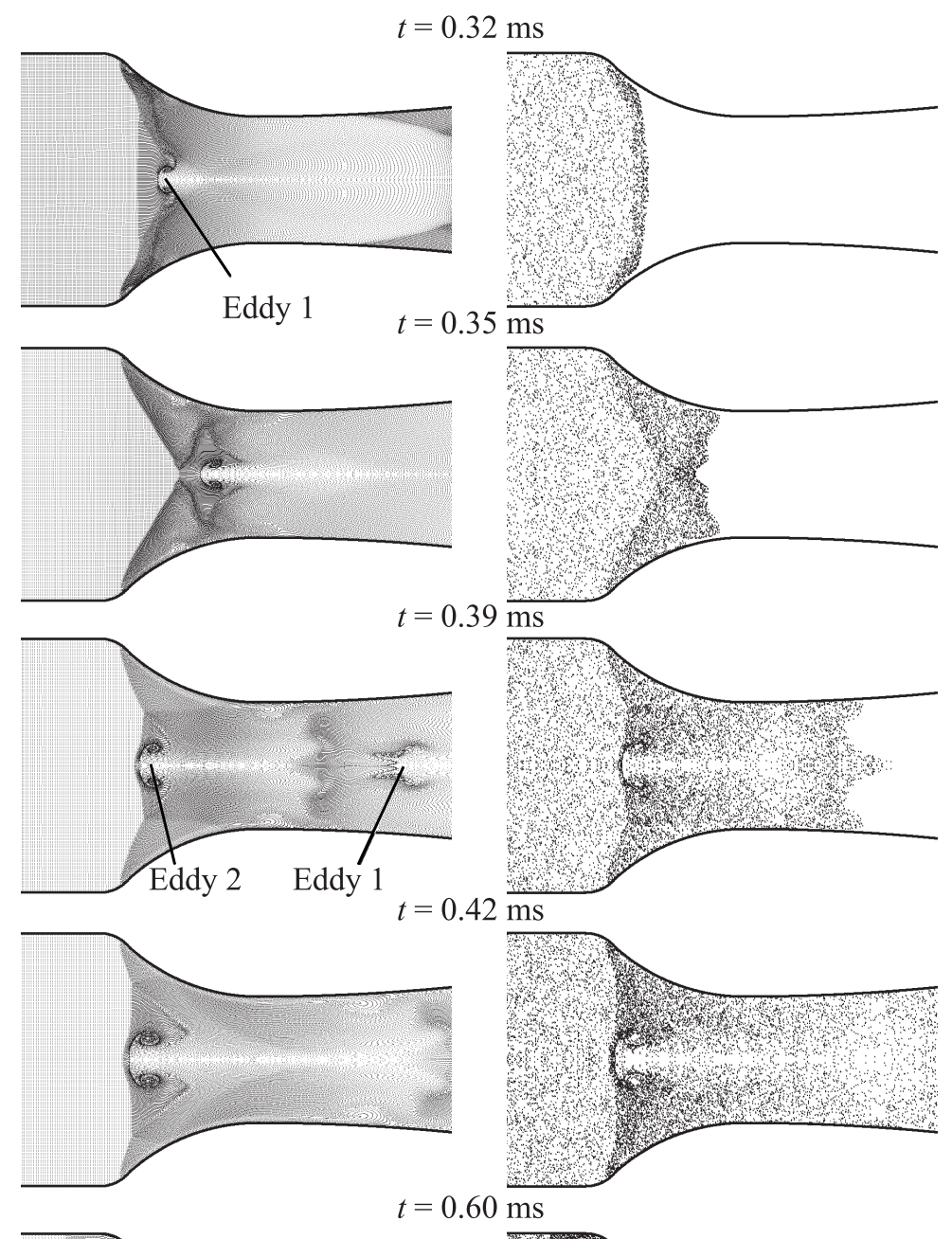

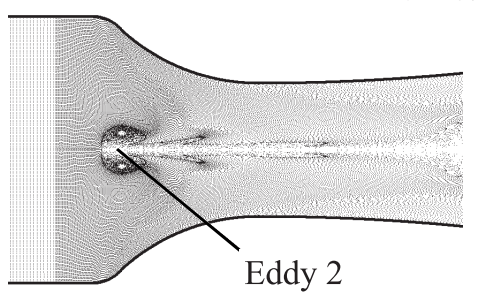

(a)

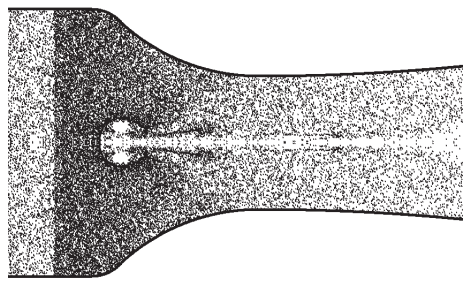

(b)

Figure 4 Instant patterns of massless particles-markers $(a)$ and dispersed particles of diameter $d_{\mathrm{p}}=0.15 \mu \mathrm{m}(b)$ in the nozzle throat at different time $t$ 
the complex shock wave structure (CD is seen in Figs. $2 d$ and $2 e$ ). During this process, initially plane CD takes a very complex configuration which is clearly seen in patterns of massless particles-markers shown in Fig. 3 (markers were distributed uniformly in the tunnel at $t=0$ ). The supersonic cocurrent flow behind PSW (the Mach number $\mathrm{M} \approx 1.3$ ) at the exit of the throat decelerates because the intensity of PSW decreases when it propagates in the divergent part of the nozzle. At the same time, the flow developing behind $\mathrm{CD}$ accelerates in the divergent part from $M \approx 1$ in the throat up to hypersonic speed $(M \approx 6)$ and impinges the much more slower flow behind PSW. As a result the secondary shock wave (SSW) is formed (see Fig. 3) which propagates upstream (to the left in the figure), but drifts by the flow towards the test section (to the right in the figure).

In the course of irregular interaction of shock waves in the throat, slip lines issuing from triple points twist, forming ring eddies, two of which are very strong. They have an extremely substantial effect on the motion of fine particles. The process of initiating, developing and drifting of these two eddies is demonstrated in the left column of Fig. 4. The second eddy forms behind the reflected shock wave (RSW) propagating upstream to the end of the high-

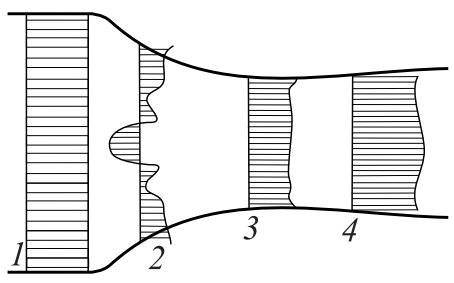

(a)

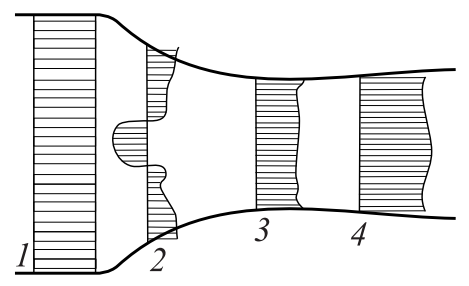

(b)

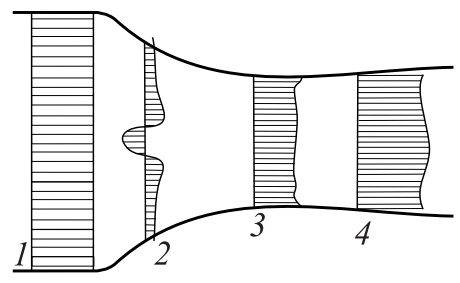

(c)

Figure 5 Profiles of $x$-component of the gas velocity in cross sections $1-4$ of the nozzle: (a) $t=0.390 \mathrm{~ms}: 1-$ velocity at the axis of symmetry $=1124.3 \mathrm{~m} / \mathrm{s} ; 2--559.7$; $3-755.2$; and $4-$ velocity at the axis of symmetry $=1294.5 \mathrm{~m} / \mathrm{s} ;(b) t=0.420 \mathrm{~ms}$ : 1 - velocity at the axis of symmetry $=1073.1 \mathrm{~m} / \mathrm{s} ; 2--436.6 ; 3-651.0$; and $4-$ velocity at the axis of symmetry $=1066.9 \mathrm{~m} / \mathrm{s}$; and $(c) t=0.600 \mathrm{~ms}: 1$ - velocity at the axis of symmetry $=874.1 \mathrm{~m} / \mathrm{s} ; 2--333.7 ; 3-552.6$; and $4-$ velocity at the axis of symmetry $=760.1 \mathrm{~m} / \mathrm{s}$ 
pressure chamber. It remains in the throat for a long time (about $0.4 \mathrm{~ms}$ ) and then drifts downstream. The lifetime of eddies depends, of course, on the actual gas viscosity. In the present study, the viscosity was not taken into account (the Euler equations were used), and the dissipation of eddies was caused by the "numerical method viscosity" which is greater than actual viscosity but still rather small. The question about eddies lifetime is open and must be the subject of further investigation. In addition to the patterns of massless particles-markers, the gas velocity profiles at different cross sections are presented in Fig. 5. As is seen, the velocity near the axis in the vicinity

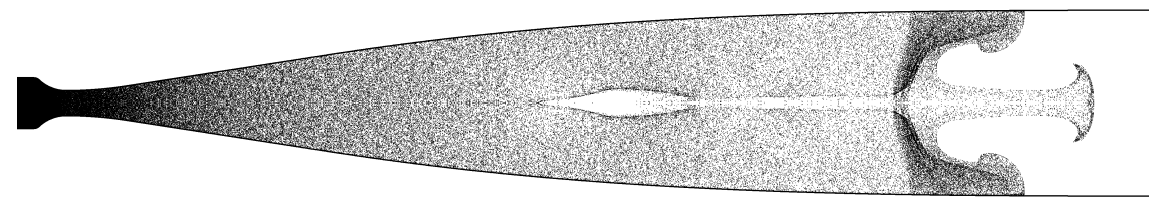

(a)

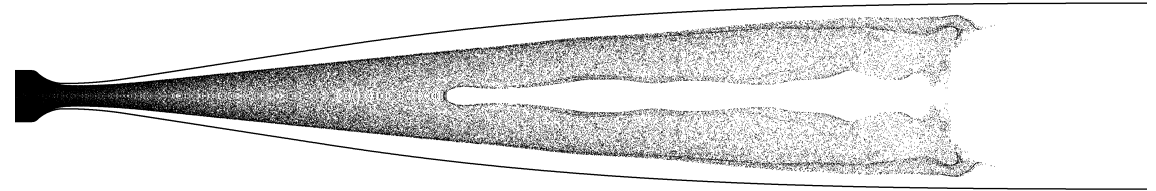

(b)

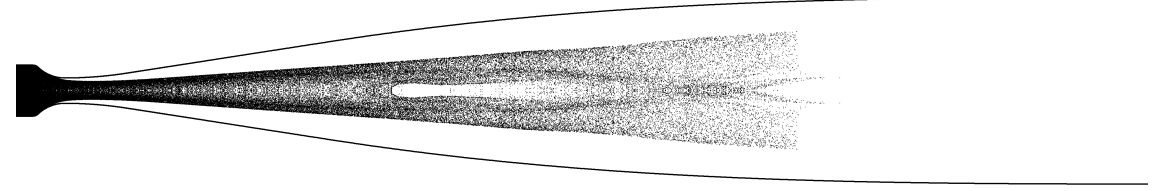

(c)

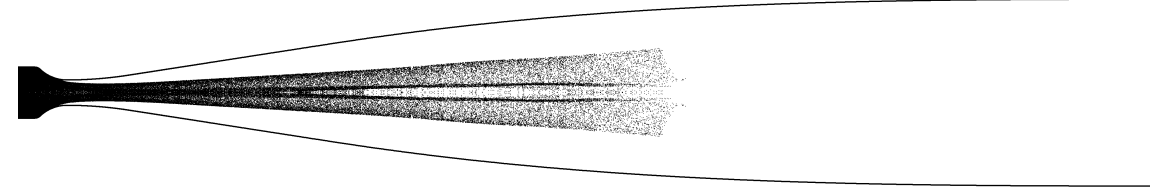

(d)

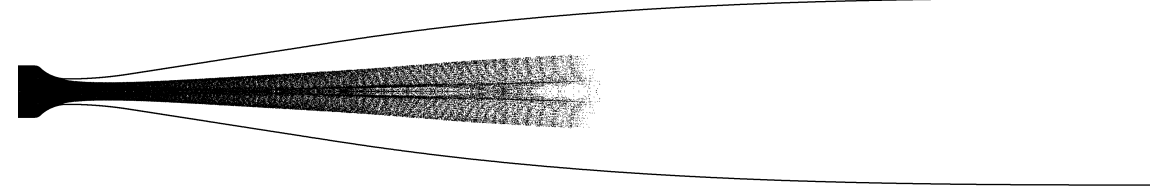

(e)

Figure 6 Instant patterns of dispersed particles of different sizes in the nozzle at time $t=1.8 \mathrm{~ms}:(a) d_{\mathrm{p}}=0.15 \mu \mathrm{m} ;(b) 5 ;(c) 10 ;(d) 15 ;$ and $(e) d_{\mathrm{p}}=20 \mu \mathrm{m}$ 
of the second eddy is opposite to the direction of the main flow during some time interval. This means that the eddy location in the throat remains constant for this interval. Eddies in the throat affect the behavior of particles, especially of the fine ones. The right column of Fig. 4 represents instant patterns of fine particles $\left(d_{\mathrm{p}}=0.15 \mu \mathrm{m}\right)$ which practically follow the carrier gas flow. As a result, the particle-phase flow structure becomes strongly nonuniform.

The particle-phase flow field depends on the particle size. Fine particles follow the carrier gas flow, whereas coarse particles, being more inertial, collide with the walls of the convergent part of the nozzle, rebound from them and form thin layers of high particle concentration. Particle-phase flow patterns of different sizes in the developing unsteady flow are shown at the same instant $t=1.8 \mathrm{~ms}$ in Fig. 6. The effect of near-axis ring eddies on the flow structure is seen in all pictures, however this effect, as can be expected, decreases with the particle size. The forward front of the particle cloud has a complex configuration which is far from planar. The space particle distribution near the front is nonuniform.

For a quasi-steady-state flow regime in the nozzle, the particles' trajectories in the throat are shown in Fig. 7. It is clearly seen that the reflected particles are focused into thin layers of high particle concentration. The profiles of the particle volume fraction (they are shown to the right from pictures with trajec-

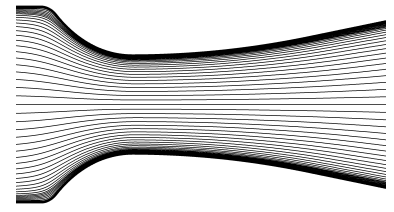

(a)

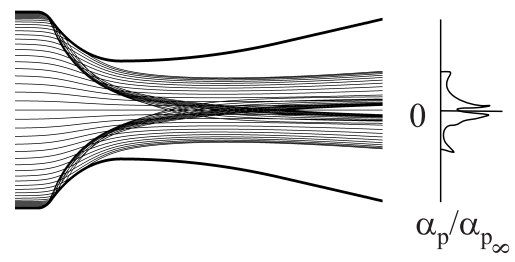

(c)

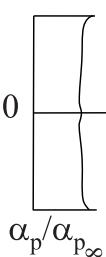

$\alpha_{\mathrm{p}} / \alpha_{\mathrm{p}_{\infty}}$

a)

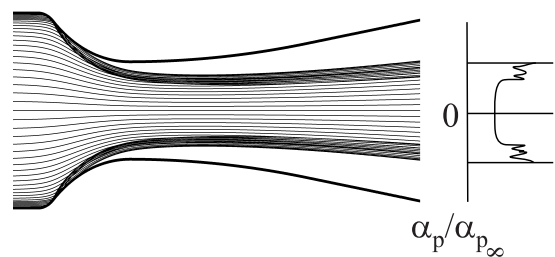

(b)

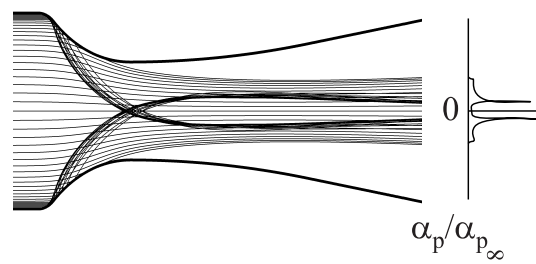

(d)

Figure 7 Trajectories of particles of different sizes in the nozzle throat in the quasisteady-state flow regime, and the corresponding profiles of the relative particle volume fraction at the right cross section: $(a) d_{\mathrm{p}}=0.15 \mu \mathrm{m} ;(b) 10 ;(c) 20$; and $(d) d_{\mathrm{p}}=40 \mu \mathrm{m}$ 
tories) demonstrate the strong nonuniformity of the particle distribution across the nozzle for all except the smallest $\left(d_{\mathrm{p}}=0.15 \mu \mathrm{m}\right)$ particle sizes. Note that these profiles are given in different scale because the maximal value of $\alpha_{\mathrm{p}} / \alpha_{\mathrm{p} \infty}$ depends substantially on the particle diameter: the maximum of $\alpha_{\mathrm{p}} / \alpha_{\mathrm{p} \infty}$ is $0.15,0.8,5.0$, and 11.0 for $d_{\mathrm{p}}=0.15,10,20$, and $40 \mu \mathrm{m}$, respectively. The same nonuniformity occurs at the entrance of the test section. This salient feature of a particle-phase nozzle flow was not taken into account in experiments [9] in which a model was set in the test section not only at the axis, but also at $41 \mathrm{~mm}$ from the axis.

Very fine particles $\left(d_{\mathrm{p}}=0.15 \mu \mathrm{m}\right)$ follow the carrier gas streamlines and their velocity lag at the entrance of the test section is less than $0.1 \mathrm{~m} / \mathrm{s}$. For larger diameter of particles $d_{\mathrm{p}}=1,5,10,20$, and $40 \mu \mathrm{m}$, the lag is $1,22,77,162$, and $250 \mathrm{~m} / \mathrm{s}$, respectively. Contrary to the particle distribution across the nozzle, the profiles of the velocity lag are almost uniform for all particle sizes.

\subsection{Flow in the Test Section}

When the primary shock wave (Fig. $8 a$, the Mach number of cocurrent flow is $\mathrm{M} \approx 1.6$ ) reaches the model in the test section, it diffracts on the model by forming a primary bow shock (Fig. $8 b$ ) which later is being deformed in the transitional process of interaction with the strongly disturbed cocurrent flow between the primary (PSW) and the secondary (SSW) shock waves (Figs. $8 c-$ $8 e$ ). And only after it passes the quasi-steady-state flow of the carrier gas with $\mathrm{M}=6.0$ begins (Fig. $8 f$ ). The flow parameters at the axis of the nozzle exit as functions of time are shown in Fig. 9. As is seen, the duration of the transitional process is about $7 \mathrm{~ms}$. But even after this process ends, all flow parameters, except the Mach number, continue to vary slowly with time. The pressure varies very weakly, whereas the variation of the speed of flow and, particularly, the density, is rather appreciable. It should be noted that the strictly steady-state flow regime of the carrier gas in the test section cannot be reached in a setup studied here. The reason is that the flow parameters at the nozzle entrance are not constant; they vary weakly but continuously. The fact is that these parameters are being formed as a result of the interaction of the reflected shock wave moving to the end of the high-pressure chamber (see Fig. $2, t=0.6 \mathrm{~ms}$ ) with the rarefaction wave initiated by diaphragm opening (at $t=0)$ and propagating in the same direction. The quasi-steady-state regime is completed at time $t=23.47 \mathrm{~ms}$, when the front of the reflected rarefaction wave comes to the test section. The duration of quasi-steady-state flow of the carrier gas over the model is about $16(=23-7) \mathrm{ms}$. The instant of the beginning $(t=7 \mathrm{~ms}$ ) is chosen on the condition that variations of all flow parameters during this regime do not exceed $5 \%$ of their values at the end (at $t=23.47 \mathrm{~ms})$. 


\section{$\mathrm{M}$}

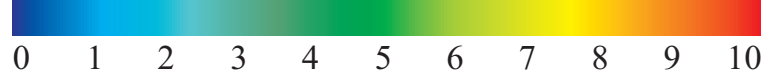

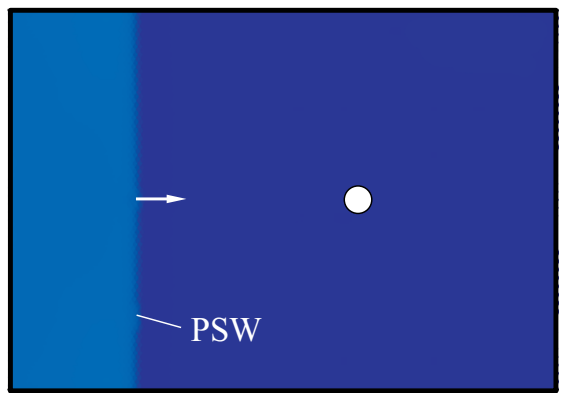

(a)

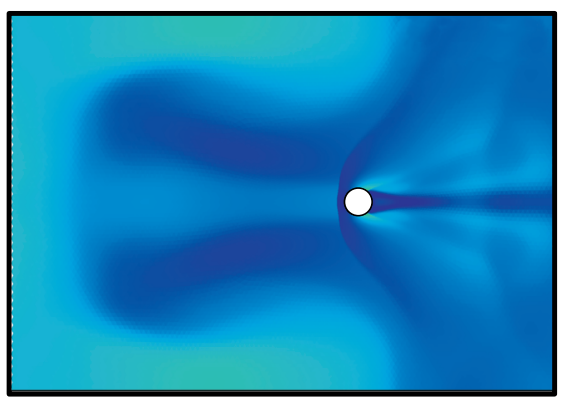

(c)

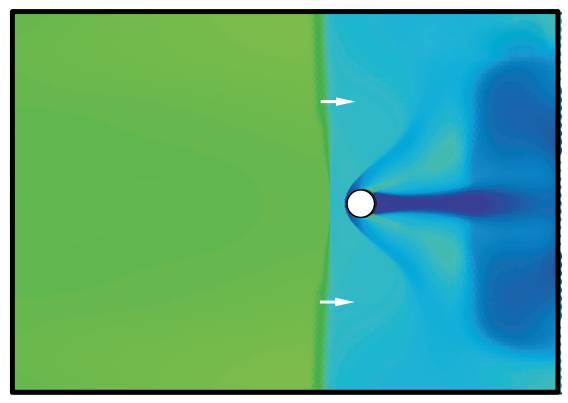

(e)

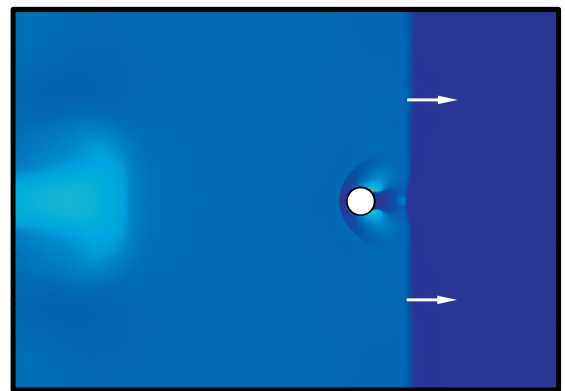

(b)

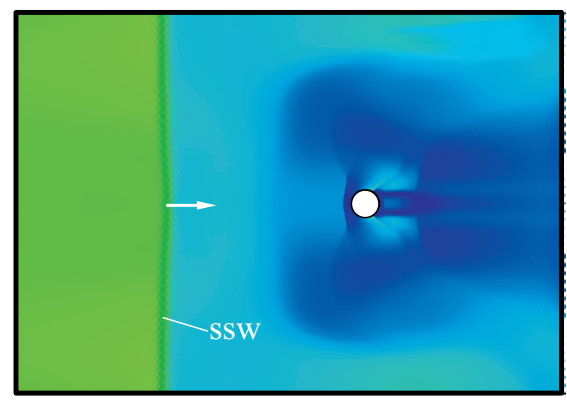

(d)

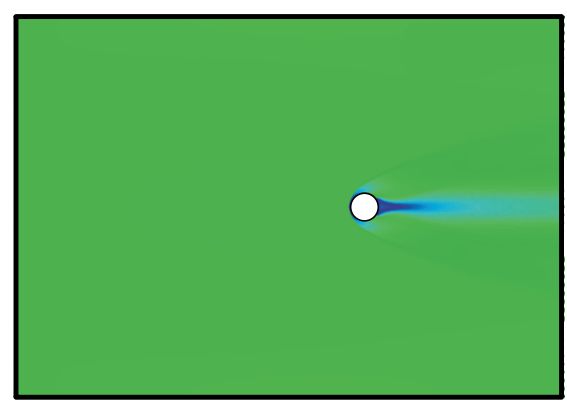

$(f)$

Figure 8 Field of Mach number in the test section at different time $t:(a) 1.8 \mathrm{~ms}$; (b) 2 ; (c) 2.4; (d) 2.6; (e) 2.8; and (f) $4.8 \mathrm{~ms}$. 

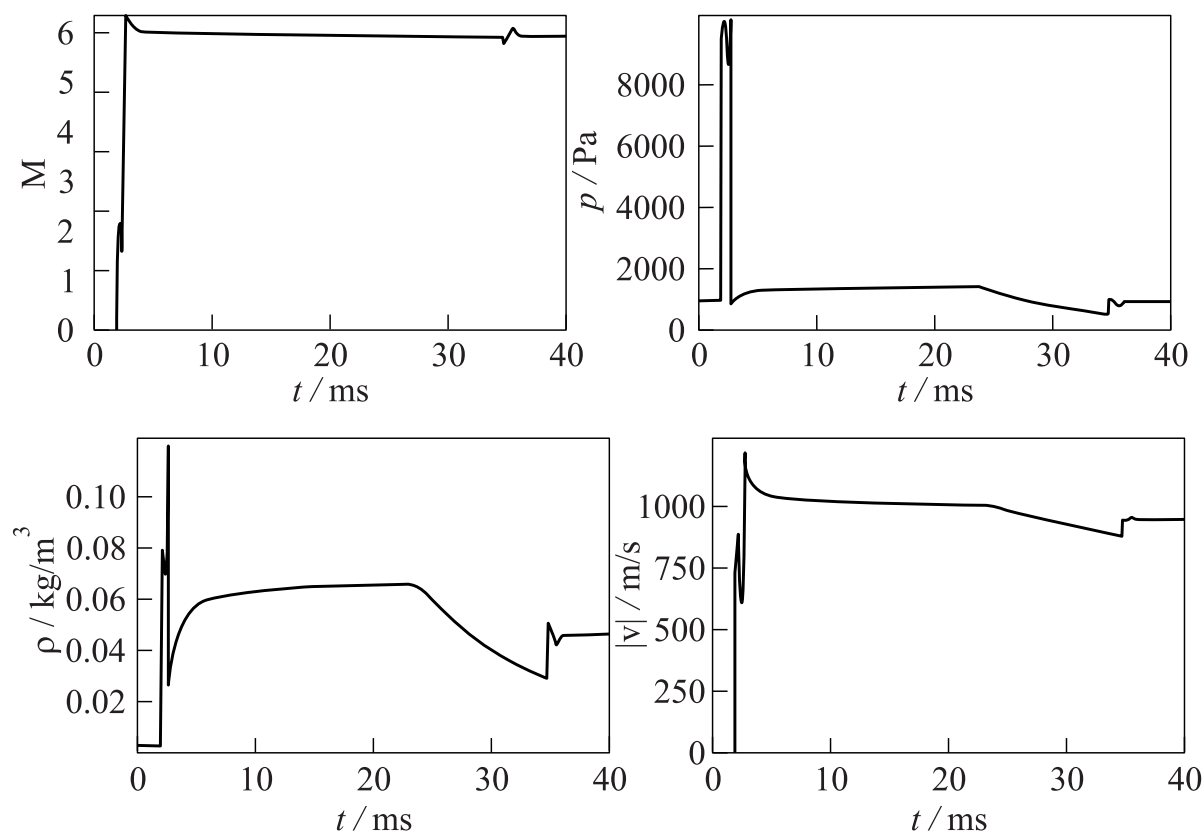

Figure 9 Gas parameters in the test section (in front of the model) as the functions of time

The shock wave traveling into the high-pressure chamber (RSW in Fig. 3) reflects from the end and moves back to the nozzle throat. Then it diffracts on the throat walls, and two shock waves, passed through the throat and reflected from it, are formed similar to the situation described above. The passed shock wave reaches the test section, and the second quasi-steady-state flow regime is realized from $t=35.81 \mathrm{~ms}$ (see Fig. 9). In this regime, the Mach number is 5.92 that is very close to the designed value, but the flow speed, the pressure and the density are less by $6.2 \%, 24.7 \%$, and $33.7 \%$, respectively, than the previous quasi-steady-state values of these parameters.

Because of the velocity lag, the particle front reaches the model later than the contact discontinuity which represents the forward front of gas initially located in the high-pressure chamber. The time delay depends on the particle size: the larger particles, the larger delay. This means that the time duration of a quasi-steady-state flow regime of a gas-particle mixture is less than that of a flow of a gas alone. Also, the particle distribution across the nozzle exit is strongly nonuniform, except very fine particles (see profiles of the relative particle volume fraction in Fig. 7). Plots of the relative particle volume fraction in the test section as a function of time for different particle sizes and different 


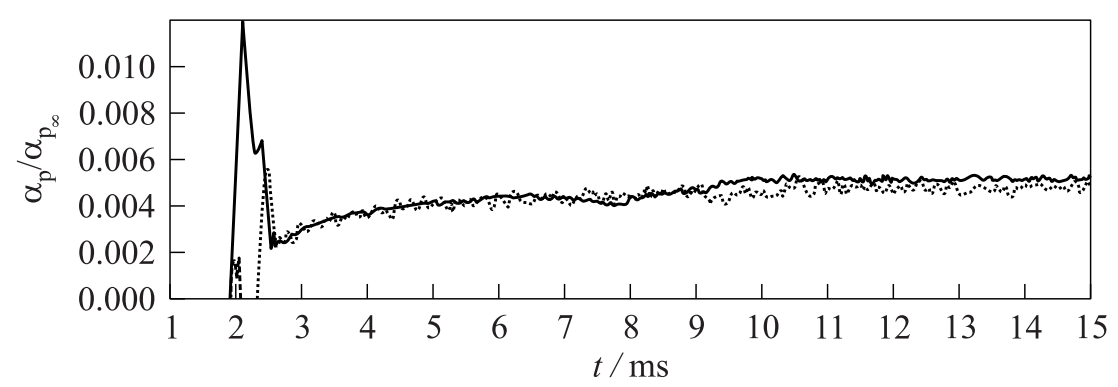

(a)

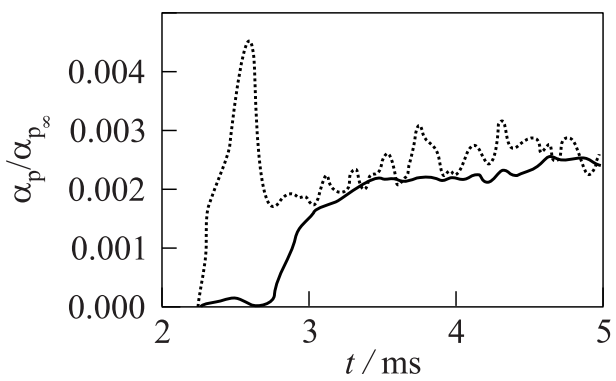

(b)

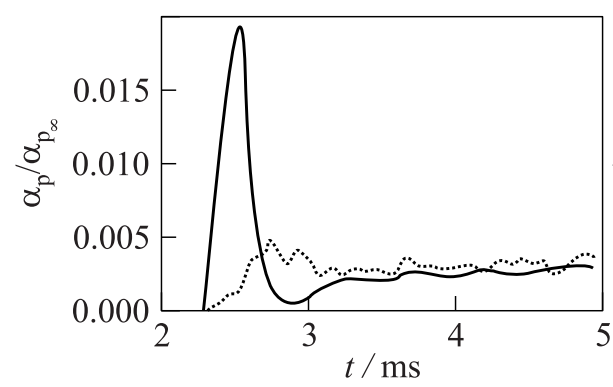

(c)

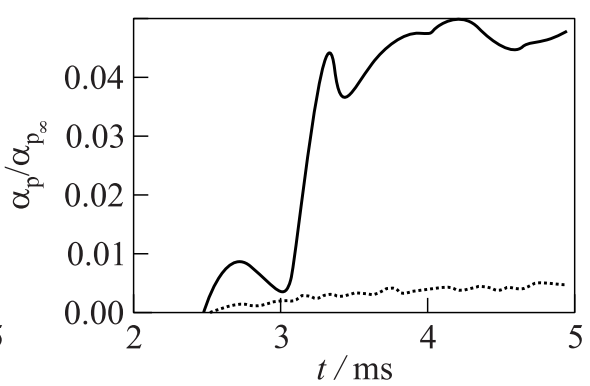

(d)

Figure 10 The relative particle volume fraction in the test section (in front of the model) as a function of time: solid lines correspond to the axis of symmetry and dotted lines correspond to the distance of $41 \mathrm{~mm}$ from the axis of symmetry: $(a) d_{\mathrm{p}}=0.15 \mu \mathrm{m}$; (b) 5 ; (c) 10; and $(d) d_{\mathrm{p}}=15 \mu \mathrm{m}$

distances from the axis are shown in Fig. 10. Solid and dotted lines correspond to the axis of symmetry and the distance of $41 \mathrm{~mm}$ from the axis, respectively. It is seen that the transitional process of going into the quasi-steady-state flow regime for the dispersed phase depends strongly on both the particle size and the distance from the axis. Slight oscillations in the particle concentration are caused, in the authors' opinion, by the insufficient number of simulated par- 
ticles in grid cells, although the number of particles taken in calculations was about 2 million. It should be noted that similar oscillations were observed in experiments [9]. As is seen from the figure, the jump of the particle concentration in time is inherent for particles of all sizes considered. Duration of the transitional process in a particle-phase flow depends on the length of the area of nonuniform particle distribution in $x$-direction. This length is greater for the most fine particles (see Fig. $6 a$ ), and for these particles the duration is the greatest (about $8 \mathrm{~ms}$, see Fig. 10a). For larger particles, the duration is much less (approximately 2-3 ms for $d_{\mathrm{p}}=5,10$, and $15 \mu \mathrm{m}$ (see Fig. 10). Duration of the quasi-steady-state flow regime for the dispersed phase for different particle sizes is correspondingly shorter (it is about $13 \mathrm{~ms}$ for particles of diameter $d_{\mathrm{p}}=0.15 \mu \mathrm{m}$ and about $16 \mathrm{~ms}$, just as for the carrier gas flow, for $d_{\mathrm{p}}=5,10$, and $15 \mu \mathrm{m})$.

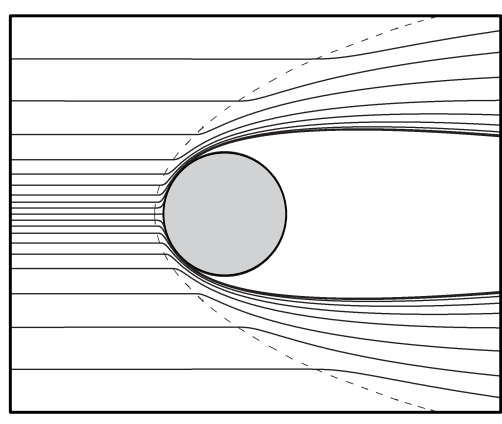

(a)

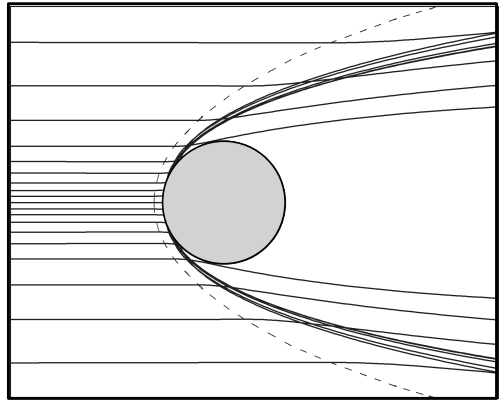

(b)

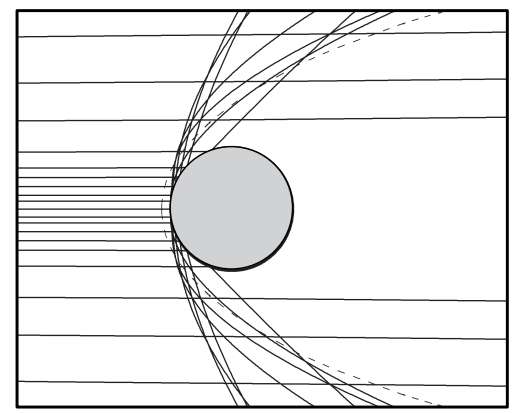

(c)

Figure 11 Particle trajectories near the model (sphere) in the quasi-steady-state flow regime with the Mach number $\mathrm{M}=6.0$; dashed curves show the positions of the bow shock wave: $(a) d_{\mathrm{p}}=0.15 \mu \mathrm{m} ;(b) 1$; and $(c) d_{\mathrm{p}}=15 \mu \mathrm{m}$ 
A pattern of particle trajectories near the model depends strongly on the particle size (more exactly, on the Stokes number, which is defined as a ratio of the particle velocity relaxation length to the characteristic length of the flow). Patterns of trajectories for different particle sizes are shown in Fig. 11. Particles of the diameter of $0.15 \mu \mathrm{m}$ move over the model (sphere) without collisions with its surface. Particles of the diameter of $1 \mu \mathrm{m}$ collide with the surface and bounce from it, but move further inside the shock layer. Bounced particles of the diameter $d_{\mathrm{p}} \gtrsim 5 \mu \mathrm{m}$ fly off the shock layer through the bow shock wave. For coarse particles, the flow pattern becomes very complex because of multiple intersections of bounced particles' trajectories.

\section{CONCLUDING REMARKS}

The feasibility of a shock tunnel for experimental study of a hypersonic dusty gas flow over bodies is determined by three factors: $(i)$ the duration of the quasi-steady-state regime in the test section; $(i i)$ the degree of nonuniformity in particles' distribution across the test section; and (iii) the magnitude of the particle velocity lag behind the carrier gas.

In the present study, an unsteady dusty gas flow in the shock tunnel UT$1 \mathrm{M}$ TsAGI was simulated from the instant of diaphragm opening to the end of the quasi-steady-state flow regime in the test section. It was found that the flow structure of the carrier gas is very complex. The primary shock wave (see PSW in Fig. 2a) diffracts on the nozzle throat with numerous regular and Mach reflections and interactions of arising transverse shock waves. It results in formation of strong ring eddies. The initially planar contact discontinuity (see CD in Fig. 2e) becomes very curved when it passes through the throat and moves in the divergent part of the nozzle (see Fig. 3). The reflected shock wave (RSW in Fig. $2 j$ ) traveling towards the end of the high-pressure chamber interacts with the rarefaction wave induced by diaphragm opening (at $t=0$ ) and propagating in the same direction, that results in the variation with the time of flow parameters at the nozzle entrance. This is the reason for that the flow in the test section never, even theoretically, reaches a strictly steady-state regime (see Fig. 9).

Because of a substantial flow nonuniformity in the longitudinal direction and the particle velocity lag, the duration of the quasi-steady-state flow regime in the test section for the dispersed phase is less than for the carrier gas. So, for the taken input data, this duration for the gas flow was about $16 \mathrm{~ms}$, whereas for fine particles, it was about $13 \mathrm{~ms}$.

The flow at the entrance of the test section is nearly uniform crosswise for fine particles and it becomes substantially nonuniform with the increase of the particle size. Coarse particles, having more inertia, collide with the walls of the 
convergent part of the nozzle and the bounced particles accumulate in thin layers of high particle concentration. Coarse particles have also a velocity lag relative to the carrier gas that makes the two-phase flow in the test section nonuniform in velocity.

All these dusty gas flow features must be taken into account when explaining or interpreting experimental results.

\section{ACKNOWLEDGMENTS}

This work was supported financially by the Russian Foundation for Basic Research through grants No. 05-08-50075 and No. 09-08-00888.

\section{REFERENCES}

1. Rakhmatulin, Kh. A., and S. S. Semenov, eds. 1962. Shock tubes. Moscow: IIL. [In Russian.]

2. Ivanov, M. Ya. 1970. Calculation of gas flow in shock tube of variable section. Izv. AN SSSR, Mekhanika Zhidkosti i Gasa. 5(3):162-66. [In Russian.]

3. Jacobs, P. A. 1991. Simulation of transient flow in a shock tunnel and a high Mach number nozzle. NASA CR 187606.

4. Saito, T., E. V. Timofeev, M. Sun, and K. Takayama. 1999. Numerical and experimental study of 2-D nozzle starting process. 22nd ISSW Proceedings. London, UK: Inperial College. Paper No. 4090.

5. Mouronval, A.-S., A. Hadjadj, A. N. Kudryavtsev, and D. Vandromme. 2002. Numerical investigation of transient nozzle flow. Shock Waves 12:403-11.

6. Saha, S., and D. Chakraborty. 2007. Numerical exploration of starting process in supersonic nozzle. Aeronautical J. Jan.:51-58.

7. Verevkin, A., and Yu. Tsirkunov. 2009. Numerical investigation of shock waves interaction in the unsteady flow after the start-up of a hypersonic shock tunnel. 27th Symposium (International) on Shock Waves Proceedings. St. Petersburg, Russia. 117-21.

8. Vasilevskii, E. B., A.V. Chirikhin, and A. N. Osiptsov. 1998. Heat transfer to a stagnation region of a blunt body in a hypersonic gas flow with an admixture of solid particles. 3rd European Symposium on Aerothermodynamics for Space Vehicles Priceedings. Noordwijk, The Netherlands. 301-7.

9. Vasilevskii, E. B., A. N. Osiptsov, A. V. Chirikhin, and L. V. Yakovleva. 2001. Heat transfer at the forward surface of a blunt body in a high-speed flow containing low-inertial particles. Inzhenerno-Fizicheskii Zhurnal 74(6):29-37. [In Russian.]

10. Henderson, Ch.B. 1976. Drag coefficients of spheres in continuum and rarefied flows. AIAA J. 14(6):707-08. 
11. Rubinow, S.I., and J.B. Keller. 1961. The transverse force on a spinning sphere moving in a viscous fluid. J. Fluid Mech. 11:447-59.

12. Oesterlé, B., and T. Bui Dinh. 1998. Experiments on the lift of a spinning sphere in a range of intermediate Reynolds numbers. Exper. Fluids 25:16-22.

13. Dennis, S. C. R., S. N. Singh, and D. B. Ingham. 1980. The steady flow due to a rotating sphere at low and moderate Reynolds numbers. J. Fluid Mech. 101:25779 .

14. Tsirkunov, Yu.M., S.V. Panfilov, and M.B. Klychnikov. 1994. Semiempirical model of impact interaction of a disperse impurity particle with a surface in a gas suspension flow. J. Eng. Phys. Thermophys. 67:1018-25.

15. Lashkov, V.A. 1991. Experimental determination of the coefficients of restitution of particles in the flow of a gas suspension in a collision against the surface. J. Eng. Phys. 60(2):154-59.

16. Godunov, S. K., A. V. Zabrodin, M. Ya. Ivanov, A. N. Kraiko, and G. P. Prokopov. 1976. Numerical solving multidimensional problems of gas dynamics. Moscow: Nauka. [In Russian.]

17. Verevkin, A., and Yu. Tsirkunov. 2006. Numerical investigation of two-phase gasparticle flow in a hypersonic shock tunnel. ECCOMAS CFD 2006 Conference Proceedings. Paper No. 211. 